\title{
Association atopic dermatitis and psoriasis in Moroccan children
}

\section{Fatima Zahra Elfatoiki, Meriem Mansouri, Hayat Dahbi Skali, Fouzia Hali, Soumia Chiheb}

Department of Dermatology, Ibn Rochd UHC, 1 quartier des Hopitaux 20000, Casablanca, Morocco

Corresponding author: Dr. Fatima Zahra Elfatoiki, E-mail: fatiza59@hotmail.fr

Sir,

Atopic dermatitis and psoriasis are the two most common immune-mediated inflammatory disorders affecting the skin in childhood. The association or overlaps of atopic dermatitis and psoriasis is not rare and sometimes poses diagnostic and management difficulties. The aim of our study is to determine clinical features in Moroccan children with psoriasis-dermatitis overlap.

A retrospective study was performed, including patients with psoriasis-dermatitis overlap, aged less than 14 years, presented to a pediatric dermatology consultation of UH Ibn Rochd of Casablanca, between January 2004 and December 2017. The diagnosis of the psoriasis-dermatitis overlap was established by the coexistence of clinical features of pediatric psoriasis (well-demarcated psoriatic plaques, guttate psoriasis and napkin Psoriasis) and atopic dermatitis criteria (UK Working Diagnostic Group) (Table. 1). We used a structured questionnaire to collect data about epidemiological, historical and clinical characteristics of patients with psoriasis-dermatitis overlap.

We collected 20 cases of psoriasis-dermatitis overlap, 12 boys and 8 girls. The mean age of disease onset was 2,16 years ( 3 months -3 years). A history of personal or familial atopy was noted in 12 patients and familial history of psoriasis in 3 patients.

Clinical features were mainly dominated by erythematosquamous lesions in 16 patients and flexural dermatitis in 14 patients (Figs. 1 and 2). Dry skin was noted in 16 patients. Pruritus in 9 patients. Fourteen children presented typical pediatric psoriasis with flexural or facial eczema (Fig. 3). The clinical features are summarized in Table 2.

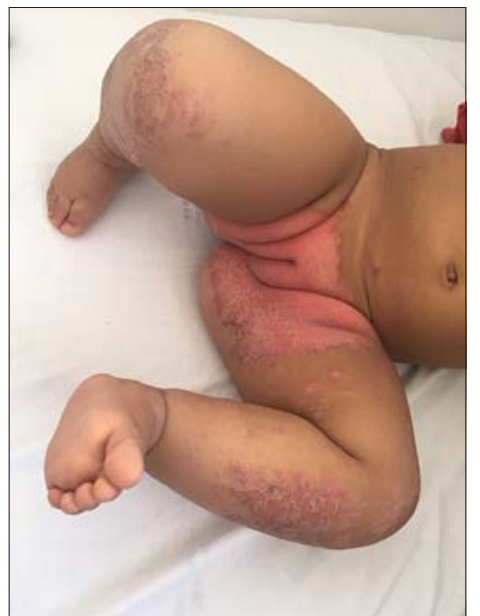

Figure 1: Nappy rash with typical atopic dermatitis of lower limbs in 18 months' infant.

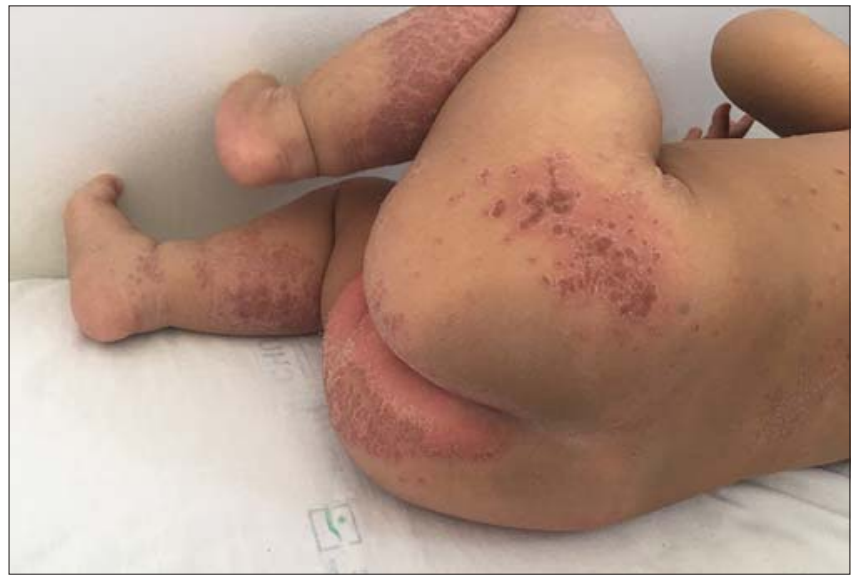

Figure 2: Nappy rash with typical atopic dermatitis of lower limbs in 18 months' infant.

For the treatment, we used topical corticosteroids in 17 cases, a topical calcipotriol- betamethasone in 3 cases and a topical tacrolimus in 5 cases.

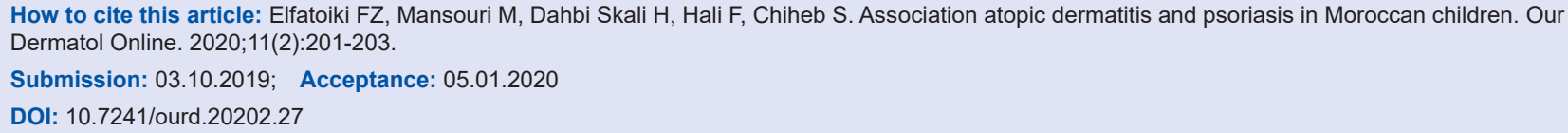




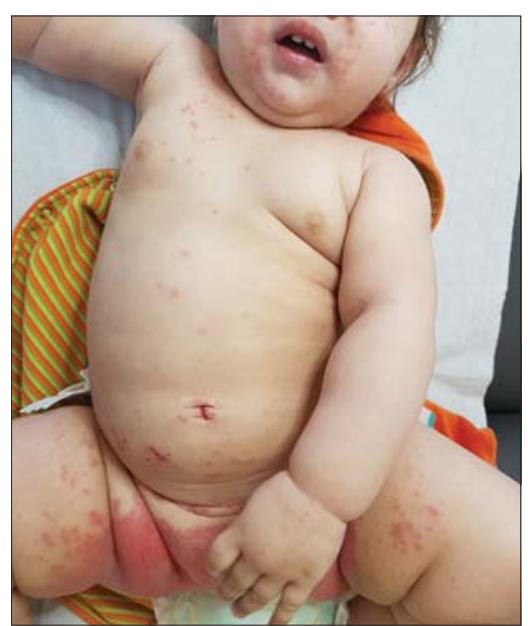

Figure 3: Nappy rash with flexural and facial dermatitis in 14 months' infant.

Table 1: The proposed diagnostic guidelines of atopic eczema [1]

Must have: An itchy skin condition (or parental report of scratching or rubbing in a child)

Plus 3 or more of the following

1-History of involvement of the skin creases such as folds of elbows, behind the knees, fronts of ankles or around the neck (including cheeks in children

under 10)

2-A personal history of asthma or hay fever (or history of atopic disease in a first-degree relative in children under 4)

3-A history of a general dry skin in the last year

4-Visible fiexural eczema (or eczema involving the cheeks/forehead and outer limbs in children under 4). 5 Onset under the age of 2 (not used if childis under 4)

Table 2: Clinical features of cutaneous involvement

\begin{tabular}{lc}
\hline Type of lesions & $\mathbf{n}(\%)$ \\
\hline Erythemato-squamous lesions & $16(80)$ \\
Palms and soles rash & $3(15)$ \\
Scaly scalp & $5(25)$ \\
Nappy rash & $8(40)$ \\
Flexural lesions & $14(70)$ \\
Periorbital lesions & $2(10)$ \\
Retro auricular lesions & $5(25)$ \\
Forehead and cheeks lesions & $7(35)$ \\
\hline
\end{tabular}

Unlike psoriasis, diagnostic criteria of atopic dermatitis have been established (UK Working Diagnostic Group) [1]. The diagnosis of psoriasis is clinical supported by family history and histology. However, cutaneous biopsy is rarely practical in children.

Atopic dermatitis and psoriasis may co-exist in the same individual. In Beer et al. study, $16.7 \%$ of atopic dermatitis patients had psoriasis and $9.5 \%$ of psoriasis patients had atopic dermatitis [2]. Maas et al. report that psoriasis in parents significantly increases the occurrence of allergic disease in their children [3].
Moreover, the risk of developing psoriasis and atopic dermatitis increased in first-degree relatives of patients. A close genetic link between the two diseases is noted from linkage studies that have identified common susceptibility loci for psoriasis and atopic dermatitis. Common genetic factors may be involved in the pathogeny of the two disorders, which leads to a production of similar inflammatory cytokines and a predominance of T-cell infiltrates in the dermis $[4,5]$. Cookson et al concluded that atopic dermatitis and psoriasis shared genoa with independent disease-specific loci and opposing effects on dermal inflammation and immunity [5].

The clinical presentation of psoriasis-dermatitis overlap shared some characteristics with childhood psoriasis. Psoriasis-dermatitis children globally appear to have more in common with children with psoriasis than those with atopic dermatitis. These young patients most often presented as typical childhood psoriasis with the addition of typical flexural atopic dermatitis and a family history of atopy or psoriasis [6]. Our study showed again that children with psoriasis-dermatitis overlap had clinical features that characterize childhood psoriasis associated with flexural or facial dermatitis. All patients had a good response to topical corticosteroids, topical calcipotriol - betamethasone and topical tacrolimus.

In conclusion, patients with psoriasis -dermatitis overlap appear to be closer clinically to patients with psoriasis. Psoriasis- dermatitis overlap responds well to topical corticosteroids. There are few studies in the literature about psoriasis - dermatitis overlap, and further studies are required to characterize this condition.

\section{Statement of Informed Consent}

Informed consent was obtained from all patients for being included in the study.

\section{REFERENCES}

1. Williams HC, Burned PGJ, Pembroke AC, Hay RJ. The U.K. working party's diagnostic criteria for atopic dermatitis. III. Independent hospital validation. Br. J. Dermatol. 1994;131:406-16.

2. Beer WE, Smith AE, Kassab JY, Smith PH, Rowland Payne CM. Concomitance of psoriasis and atopic dermatitis. Dermatology. 1992;184:265-70. Erratum in: Dermatology. 1992;185:236.

3. Maas T, Nieuwhof C, Passos VL, Robertson C, Boonen A, Landewé RB, et al. Transgenerational occurrence of allergic disease and autoimmunity: general practice-based epidemiological research. Prim Care Respir J. 2014;23:14-21. 


\section{www.odermatol.com}

4. Wilsmann-Theis D, Hagemann T, Jordan J, Bieber T, Novak N, Bieber T, Novak N. Facing psoriasis and atopic dermatitis: are there more similarities or more differences? Eur. J. Dermatol. 2008;18:172-80.

5. Cookson WO, Ubhi B, Lawrence R, Abecasis GR, Walley AJ, Cox HE, et al. Genetic linkage of childhood atopic dermatitis to psoriasis susceptibility loci. Nat. Genet. 2001;27:372-3.

6. Kapila S, Hong E, Fischer G. A comparative study of childhood psoriasis and atopic dermatitis and greater understanding of the overlapping condition, psoriasis-dermatitis. Australas J Dermatol. 2012;53:98-105.

Copyright by Fatima Zahra Elfatoiki, et al. This is an open-access article distributed under the terms of the Creative Commons Attribution License, which permits unrestricted use, distribution, and reproduction in any medium, provided the original author and source are credited.

Source of Support: Nil, Conflict of Interest: None declared. 